\title{
Control for Reheat Steam Temperature of Power Plant Based on Particle Swarm Optimization
}

\author{
Zhang Hongtao ${ }^{1}$,Hu Hongli ${ }^{2}$, Xu Xinhang ${ }^{1}$, Liu Yonghong ${ }^{1}$, Wang Lei ${ }^{1}$, Pan Junwei ${ }^{1}$ \\ 1. Hebei Electric Power Research Institute \\ 2. North Design\&Research Institue
}

\begin{abstract}
The model of reheat steam temperature of power plant has characteristics such as large delay and large inertia, the traditional PID control is difficult to achieve good results. his paper presents a new PSO with random acceleration factors and verifies its validation, also, this paper presents a new control method, uses the modified PSO to identify the model, tunes parameters SIMTH-PID control based on particle swarm optimization and the results of identification. The simulation results show that the method is more effective than the traditional PID control.
\end{abstract}

KeyWords-Particle swarm optimization, Reheat steam temperature, Identification

\section{INTRODUCTION}

In order to improve the economy of thermal cycling , large coal-fired units generally use reheat system. Large boiler reheater works at high temperature near the limit of its metal pipes, over high temperature will decrease the safety of the metal pipe ,over low temperature will decrease economy, therefore, the reheat steam temperature is one of the most important parameters. [1]

Reheat steam temperature is usually regulated by gas baffle, the model has obvious characteristics of large delay and large inertia, the traditional pid control is difficult to achieve good control results. SMITH control is the solution as the control of large delay[2], which can eliminate some extent delay and greatly improve the quality of control. In process control, combined of PID SMITH control is widely used .This method involves some parameters settings of SMITH control module and the PID control. In this paper, a method of tuning the above parameters was proposed.

\section{The CONTROL OBJECT OF REHEAT STEAM TEMPERATURE}

In usually, gas baffle is used to control the reheat steam temperature of thermal power plant, and spray water is used when the temperature is too high . Reheat steam flow through the reheater in the seperation gas pass and the reheater in furnace, it was heated by heat transition of gas metal - steam . Reheat steam temperature refers to the last reheater (High temperature reheater in furnace) outlet steam temperature .Gas baffle is installed in the seperation gas pass, changing the gas baffle's opening, can change the the reheater gas flow, thus chang the reheat steam temperature of reheater outlet in the seperation gas pass,and ultimately change the reheat steam temperature.[2]

This shows, the control object of reheat steam temperature has a large delay.The heat transition of gas - metal - steam has large itertia.So, the control object of reheat steam temperature has obvious characteristics of large delay and large inertia.Usually,we describe it as secondorder plus delay system:

$$
G(s)=\frac{K e^{-\tau s}}{\left(T_{1} s+1\right)\left(T_{2} s+1\right)}=G_{p}(s) * e^{-\tau s}
$$

The Control Method of Reheat Steam Temperature

Combined of PID and SMITH control is widely used in reheat steam temperature control, the control method is as follows:

$G_{C}(S)$ is PID controller which is generally taken to PI control, $G_{c}(s)=k+1 / T_{I} *_{s}, R(s)$ is the opening of gas baffle, $Y(s)$ is reheat steam temperature, $G(s)$ 、 $G_{p}(s) 、 e^{-\tau s}$ means as formula (1)。

By Figure 1 and the principle of SMITH control, when $G_{p}(s)$ and $e^{-\tau s}$ match with the actual model,the effect of delay is eliminated. Combined with PID control, good control results are achieved. In this control method, there are six unknowns, they are gain $k$ and integral time $T_{I}$ of PID, the gain coefficient $K$, time constant $T_{1}, T_{2}$, delay time $\tau$ of the control object. The method of parameter setting is given as following, control object parameters are obtained by model identification, PID parameters are obtained by optimizing .

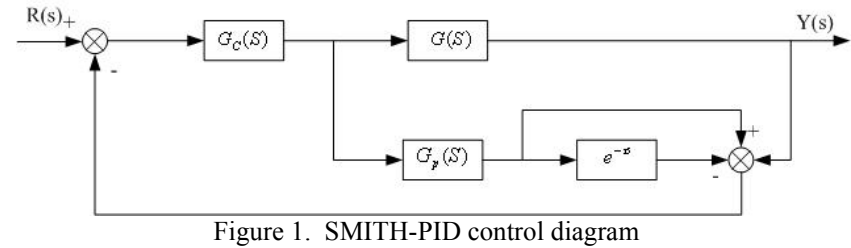

\section{PARTICLE SWARM OPTIMIZATION}

\section{A. Particle Swarm Optimization [3]}

PSO is a population-based evolutionary algorithm, using speed - location model, each particle denotes a candidate solution in D-dimensional space. D is the number of unkowns . Each particle adjusts its flight based on flight experience. The ith particle 's velocity 
$v_{i}=\left(v_{i 1}, v_{i 2}, \cdots, v_{i D}\right)$, location $x=\left(x_{i 1}, x_{i 2}, \cdots, x_{i D}\right)$, the self-optimal solution $p_{i}=\left(p_{i 1}, p_{i 2}, \cdots, p_{i D}\right)$, the global-optimal solution $p_{g}=\left(p_{g 1}, p_{g 2}, \cdots, p_{g D}\right)$. Velocity update is as follows:

$$
\begin{gathered}
v_{i d}(k+1)=w \cdot v_{i d}(k)+c_{1} r_{1}\left(p_{i d}-x_{i d}(k)\right) \\
+c_{2} r_{2}\left(p_{g d}-x_{i d}(k)\right) \\
x_{i d}(k+1)=x_{i d}(k)+v_{i d}(k)
\end{gathered}
$$

$w_{\text {is Inertia weight; }} r_{1} 、 r_{2}$ are random numbers in $[0,1] ; \quad c_{1}, c_{2}$ are acceleration factor; $k$ is iteration number。 last.

The optimal solution in D-dimensional space is found in

\section{B. General Modified PSO}

Many scholars have proposed ways to improve based on simple PSO, One of the most effective ways is variable inertia weight .The arger inertia weight $(w)$ can enhance the global serch ability of the algorithm, otherwise, the smaller $w$ can enhance the local serch ability and accelerate the iteration speed.

Literature [4] proposed a random inertia weight generation methods, the results show that the inertia weight obeying normal distribution ( $\mu$ obeys equidistribution $[0 \sim 1], \sigma=0.5$ ) has more global search ability and faster iteration speed.

\section{PSO with Random Acceleration Factor}

Base ideas of the part B, in order to maintain the diversity of groups, random acceleration factor is more effective than constant acceleration factor.

In PSO, $c_{1}$ characterizes the effect of the particles' own experience, $c_{2}$ characterizes the effect of the particles' group experience .Usually, $c_{1}=c_{2}=1$, we take $c_{1}, c_{2}$ obeying equi-distribution [1.7 2] and test the affection use Rosenbrock function as :

$$
f(x)=\sum_{i=1}^{n}\left(100\left(x_{i+1}-x_{i}^{2}\right)^{2}+\left(1-x_{i}\right)^{2}\right)
$$

The results are as follows, method 1 is the basic PSO, method 2 is the PSO with random inertia weight, method 3 is the PSO with random inertia weight and random acceleration :

Table I Test results of Rosenbrock function

\begin{tabular}{|c|c|c|c|c|}
\hline Method & $\begin{array}{c}\text { Dimensio } \\
\text { n }\end{array}$ & $\begin{array}{c}\text { Populatio } \\
\text { n size }\end{array}$ & $\begin{array}{c}\text { Convergence } \\
\text { rate }\end{array}$ & Iterations \\
\hline \multirow{2}{*}{ Method1 } & 20 & 50 & $14 / 50$ & 5192 \\
\cline { 2 - 5 } & 30 & 100 & $8 / 50$ & 6227 \\
\hline
\end{tabular}

\begin{tabular}{|c|c|c|c|c|}
\hline \multirow{2}{*}{ Method 2 } & 20 & 50 & $18 / 50$ & 4832 \\
\cline { 2 - 5 } & 30 & 100 & $15 / 50$ & 6201 \\
\hline \multirow{2}{*}{ Method 3 } & 20 & 50 & $23 / 50$ & 4662 \\
\cline { 2 - 5 } & 30 & 100 & $21 / 50$ & 5814 \\
\hline
\end{tabular}

The results show that the method 3 is better than method 1 and method 2 .

\section{Model IdENTIFICATION BASE ON PSO}

\section{A. PSO Model Identification Procedure}

PSO model identification is emplement as following: get the step response of the model (or other response), denoted by $\mathrm{y}$, the particle structure is [ $\left[\begin{array}{llll}\mathrm{K} & \tau & \mathrm{T} 1 & \mathrm{~T} 2\end{array}\right]$, each particle denotes a certain model, get the step response of the model, denoted by y', select a appropriate fitness function fitness $=f\left(y, y^{\prime}\right)$, optimize the unkowns by fitess, after many times iterate, the global-optimal solution is the result of the identification.

Step 1: Set the population size, upper and lower limits of each parameter, select $\mathrm{c} 1, \mathrm{c} 2$ and the maximum iteration number

Step 2: In accordance with [ $\left[\begin{array}{llll}K & \tau & \mathrm{T} & \mathrm{T} 2\end{array}\right]$ structure, initial population location, velocity vector, the particle's selfoptimum fitness and the global optimum fitness.

Step 3: According to the corresponding relations between the particle elements and model parameters, get the model and its step response,calculate the particle's fitness.

Step 4: Update the particle's self optinum solution and fitness, the global optimal solution and fitness.

Step 5: Update position and velocity of each particle. If its location is over limit, then reinitialize it.

Step 6: If not reach the maximum number of iterations, then go to step 3 .

\section{B. Identification of Reheat Steam Temperature}

According to experience, we set the range of parameters: $\mathrm{K} \in[0.01 \sim 100], \mathrm{T} 1, \mathrm{~T} 2 \in[0.1 \sim 100], \quad \tau \in[0 \sim 300]$, set speed limit to the difference between maximum and minimum parameters, set population size to 40 set the maximum number of iterations to 300 , and generate $\mathrm{c} 1, \mathrm{c} 2$ as the part III-B.

The delay time of the model influence SMITH control effect seriously, so in the calculation of fitness, we increase the weigh of data in the pure delay area, as:

$$
\text { fitness }=\left(\sum_{i=1}^{n} a^{*}\left(y_{i}^{\prime}-y_{i}\right)^{2}\right)^{-1}
$$

In the formula $4, \mathrm{n}$ is current number of iterations, if $\mathrm{n}<$ $\tau * 1.1, \mathrm{a}=50$; if not, $\mathrm{a}=1$.

Take a reheat temperature control object of $300 \mathrm{MW}$ unit ,step the gas damper by $5 \%$, record the reheated steam temperature response curve and data from the distribute control system (DCS). According to Section 3, we 
identify the model by Matlab, the results of identification are as follows:

$$
G(s)=\frac{0.791 * e^{-129.5 s}}{(108 s+1)(145 s+1)}
$$

It can be seen from the Figure 2, the identification curves in good agreement with the actual curve.

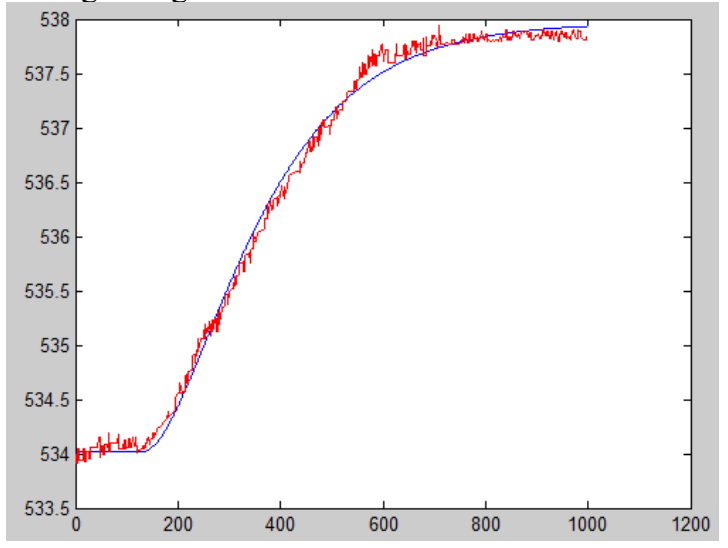

Figure 2. Identification of reheat steam temperature

\section{PID TUNING BASED ON PSO}

The same with the model identification, PID parameter setting can also be carried out by particle swarm optimization.

The expression of PID control is as follow :

$$
G_{c}(s)=k+1 / T_{I} * s
$$

In formula (7): $k$ is gain, $T_{I}$ is intergral time. At this point, optimization of parameters turn into [gain , integral time] ( $\left.\left[k T_{I}\right]\right)$. According to experience, we set the range of parameters: $\mathrm{k} \in[0.01 \sim 100]$, TI $\in$ [100 1000], set speed limit to the difference between maximum and minimum parameters, set population size to 40 set the maximum number of iterations to 300 , and generate $\mathrm{c} 1, \mathrm{c} 2$ as the part III-B.

Take the fitness function :

$$
\text { fitness }=\left(\int t *|(e(t))| d t\right)^{-1}
$$

In formula ( 8$): t$ is the time coordinate of the step response, $e(t)$ is the difference between the setpoint and the value of reheat steam temperature.

Several tests show that the fitness hasn't many local extreme points and it can approach the global extreme points in 50 inertias, so, we make the following improvements :

Take the max inertias to 50 , and use the above method to get the best particle $\left(\left[k_{b 1} T_{I b 1}\right]\right)$; Then, take the max inertias to 50 , set the range of parameters as $k \in\left[\begin{array}{ll}0.8 & 1.2\end{array}\right]^{*} k_{b 1}, T_{I} \in\left[\begin{array}{ll}0.8 & 1.2\end{array}\right]^{*} T_{I b 1}$, use the above method again to get the final global best particle. So ,the max inertias reduced to 100 from 300 , and the best particle had high accuracy.

The flow diagram of the PID tuning based on PSO shows as Figure 3.

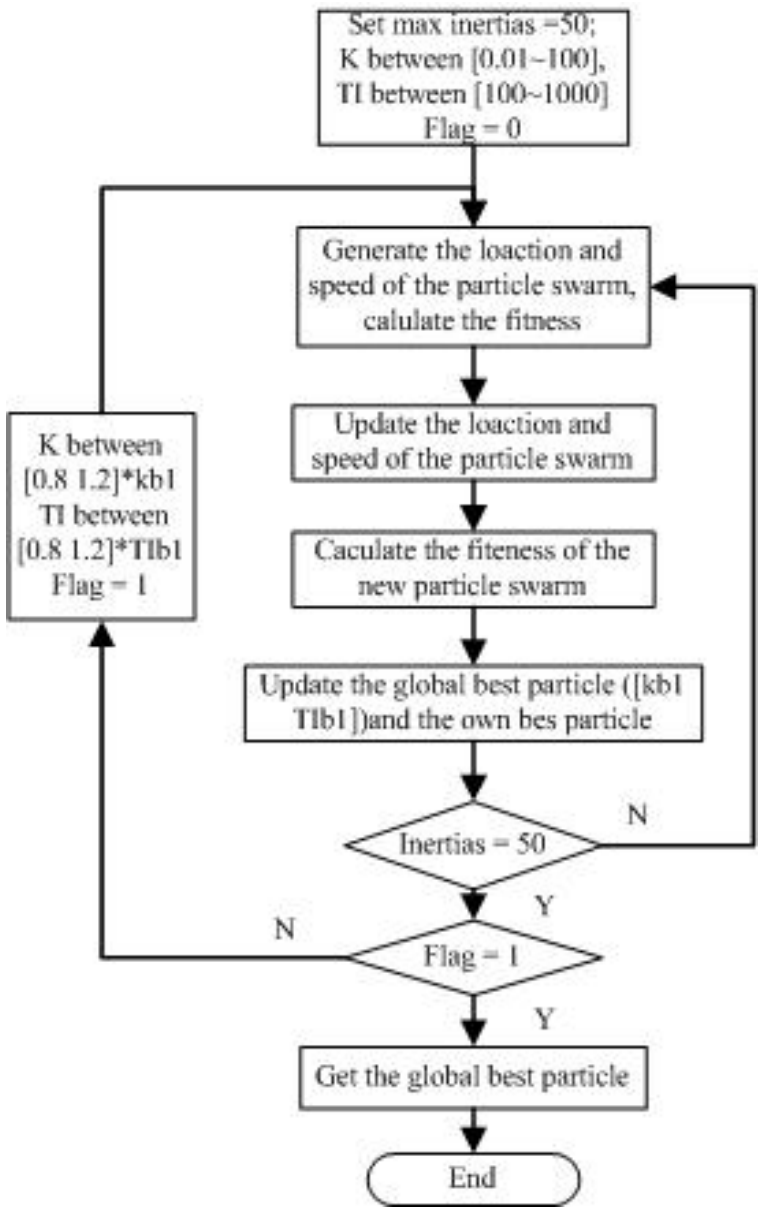

Figure 3. the produce of PID tuning based on PSO

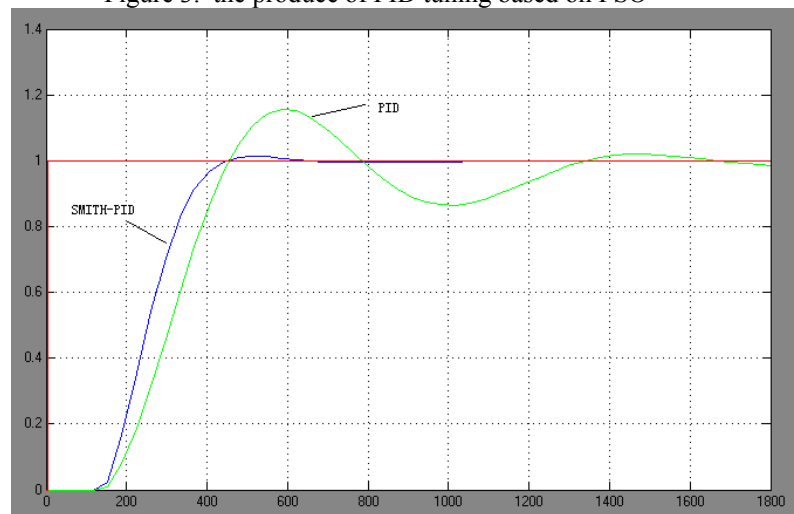

Figure 4. the control effect of SMITH-PID and PID 
Use the above method, the final global best particle is [2.132 113], $k=2.132, T_{I}=113$, the effect of control shows as Figure 4.

Compared with the pid, SMITH-PID has a faster rise time, smaller overshoot and shorter steady time.

\section{CONCLUSION}

This paper presents a modifid PSO and a control method of reheat steam temperature based on modified PSO and SMITH-PID. Simulation results show that the method of control effect is good.

\section{REFERENCES}

[1] HUAG Yong-hong,LI Nian-ping.Research on model predictive control of reheat steam temperature of large scale coal-fired units. Hunan Eectric Power[J],2007 PP:1-4

[2] ZHANG Hont-tao.Identification of Thermodynamic Process Through Model Based on Modified Particle Swarm algorithm, Thermal Power Generation[J].2010 PP:59-61

[3] Kennedy J, Eberhart R C. Particle Swarm Optimization . IEEE International Conference on Neural Networks[J] , Piscataway . 1995 PP: $1942-1948$

[4] HU Jian-xiu ,ZENG Jian-chao , A Particle Swarm Optimization Model with Stochastic Inertia Weight,Comuter Simulation [J] . 2006 PP 164-167 